\title{
New High Performance Hybrid Magnet Plates for DNA Separation and Bio-Technology Applications
}

\author{
D. Humphries, M. Pollard, C. Elkin, K. Petermann, C. Reiter, M. Cepeda
}

\begin{abstract}
A new class of magnet plates for biological and industrial applications has recently been developed at the D.O.E. Joint Genome Institute and Lawrence Berkeley National Laboratory (JGI/LBNL). These devices utilize hybrid technology that combines linear permanent magnet material and ferromagnetic material to produce significantly higher fields and gradients than currently available commercial magnet plates. These hybrid structures incorporate ferromagnetic poles that can be easily shaped to produce complex field distributions for specialized applications. The higher maximum fields and strong gradients of the hybrid structures result in greater holding forces on magnetized targets that are being processed as well as faster draw-down. Current development versions of these magnet plates have exhibited maximum fields in excess of 9000.0 Gauss $\dagger$. The design of these structures is easily scalable to allow for field increases to significantly above 1.0 tesla (10000.0 gauss).
\end{abstract}

$\uparrow$ Author's note: 11000.0 Gauss peak fields have been achieved as of January 2005.

\section{General attributes}

The core magnetic structure is shown in figure 1. It consists of an aluminum base, a permanent magnet array, a pole array and magnet retainers. An exploded view of the structure is shown in figure 2 . These hybrid structures are powered solely by permanent magnets and require no external power source. They are compact, with a footprint slightly larger than a standard micro-titer plate ${ }^{*}$ and a thickness of approximately 1 inch. They have been adapted for use with a number of different micro-titer plates and a variety of commercial liquid handling robots and other instruments including 96 and 384 channel Robbins Co. Hydra dispensers.

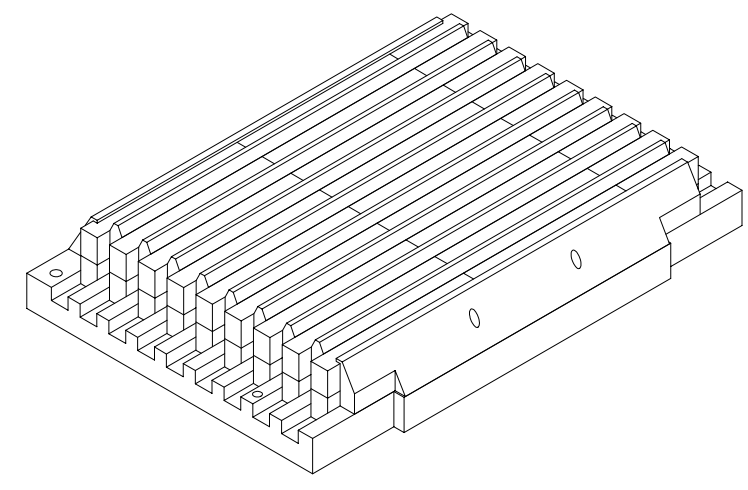

Figure 1. Hybrid magnetic structure core assembly.

\footnotetext{
* Microtiter plates are industry-standard plastic plates that conform to a standard footprint size and that incorporate 96 , 384 or 1536 wells that act as containers for various biological and chemical solutions. 


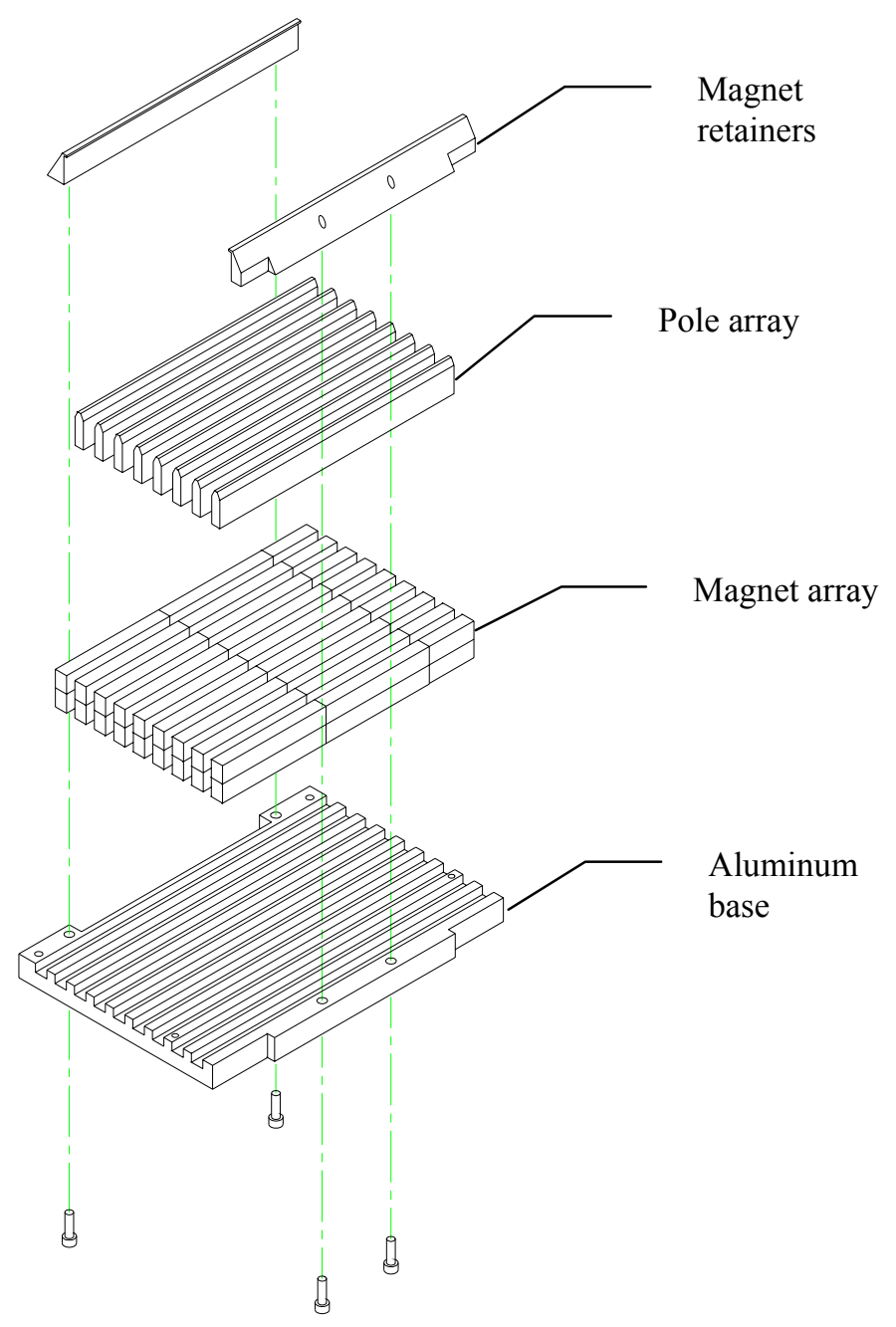

Figure 2. Exploded view of core hybrid magnetic structure.

\section{Magnetic circuit}

A cross section of the magnetic structure showing pole placement and orientations of the permanent magnet (P.M.) material is shown in figure 3. The horizontally oriented permanent magnets with alternating directions, create a large pole-to-pole scalar potential difference that results in high magnetic flux density between the upper pole tips and a corresponding, alternating polarity. The P.M. material that extends below the poles inhibits the pole-to-pole flux and results in a reduced field at the lower surfaces of the magnetic structure.

As the height of the structure is increased, the flux density in the pole tips increases up to the limiting case where the pole tips reach their saturation point. For common magnet steels this saturation point is at approximately 17 kilo-gauss. The implications are that the utilizable field levels for these magnetic structures can be close to that saturation field level. In addition, because of the near-saturation condition in the magnet poles, the field gradients (and hence, the forces on magnetized particles) can be very strong. 


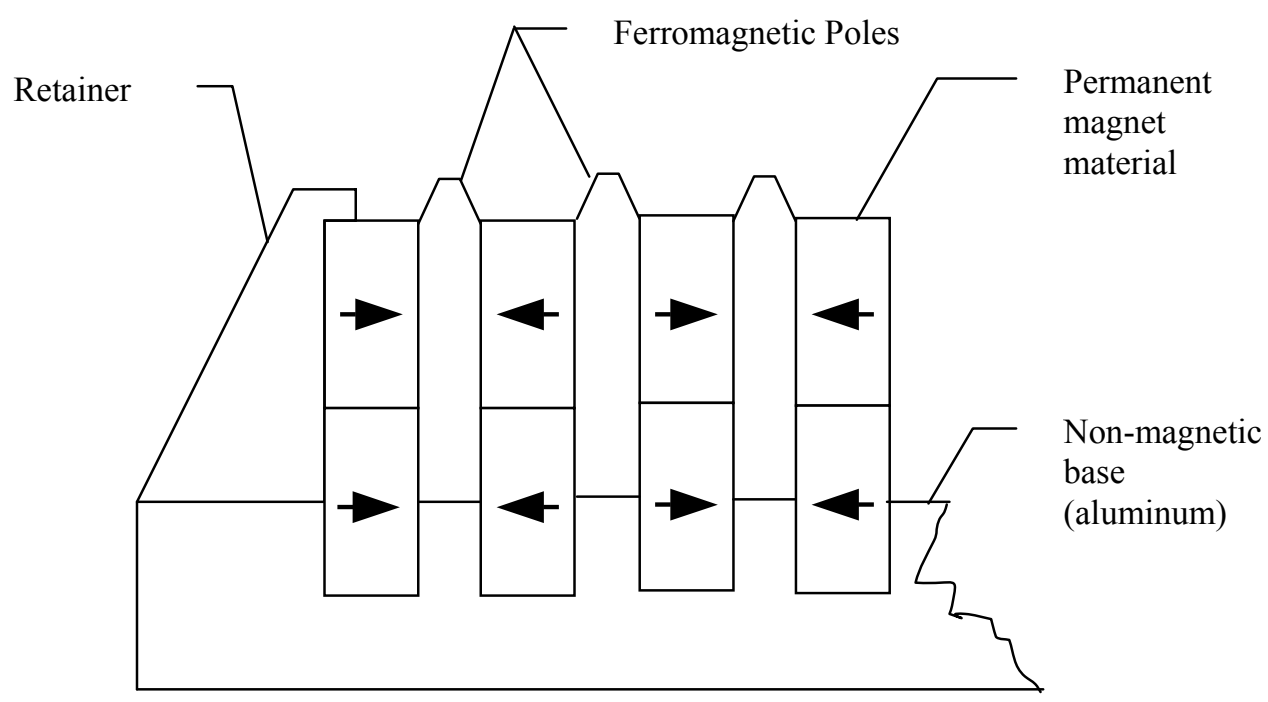

Figure 3: Cross section of magnetic structure with permanent magnet orientations.

\section{$\underline{\text { Applications }}$}

These plates are currently being used in the development of DNA purification processes for production sequencing and functional genomics at the Joint Genome Institute.

This technology is applicable to work in the broader field of functional genomics and proteomics since it can be used for selective separation of proteins from cellular and other matter. In addition, these plates can be used in high-throughput drug development and other industrial processes requiring magnetic separation of dense arrays of samples in solution.

These plates are designed to be used in conjunction with most industry standard micro-titer plate formats including 96, 384 and 1536 well plates. They may also be used for separation processes involving unpartitioned containers.

The magnetic structure is designed to act on any magnetized molecular substance in a liquid solution. It has been used to separate magnetized DNA fragments from bacterial cellular matter after plasmid DNA amplification and to separate magnetic particles from DNA that has released those particles after processing. It can be used, for example, to manipulate magnetized protein molecules for proteomics applications. It is designed to act directionally on magnetized particles by creating a fine structure of strong field gradients which match the structure of the various microtiter plate well arrays.

\section{Magnetized DNA - a specific example}

DNA is magnetized using carboxylate modified ferrite beads ranging from 0.8 to 3.0 microns in diameter. These beads are placed in solution with the DNA molecules and bind to receptor sites. After processing, the beads can be released from the DNA by means of simple hydration techniques. A specific application of magnetic bead purification for high-throughput sequencing is described in reference [2]. 


\section{$\underline{\text { Performance comparison }}$}

Relative field strengths of five different magnet plates are shown in figures 4 and 5 . Three of the magnet plates (with "LBNL" designation) were developed at JGI/LBNL. The other two magnet plates are commercially available models.

The field strengths were measured at two heights, a) less than $0.5 \mathrm{~mm}$ above the magnet surface and b) at 1 $\mathrm{cm}$ above the magnet surface. Measurements were made using a Hall effect probe.

As shown in figure 4, the hybrid magnetic structure produces fields at the magnet surface that are $70 \%$ greater than the best performing of the industry 384-well magnet plates tested. When compared to the most commonly used commercial 96-well magnet plate, the performance differential is more dramatic. The maximum fields of the hybrid are approximately $340 \%$ greater.

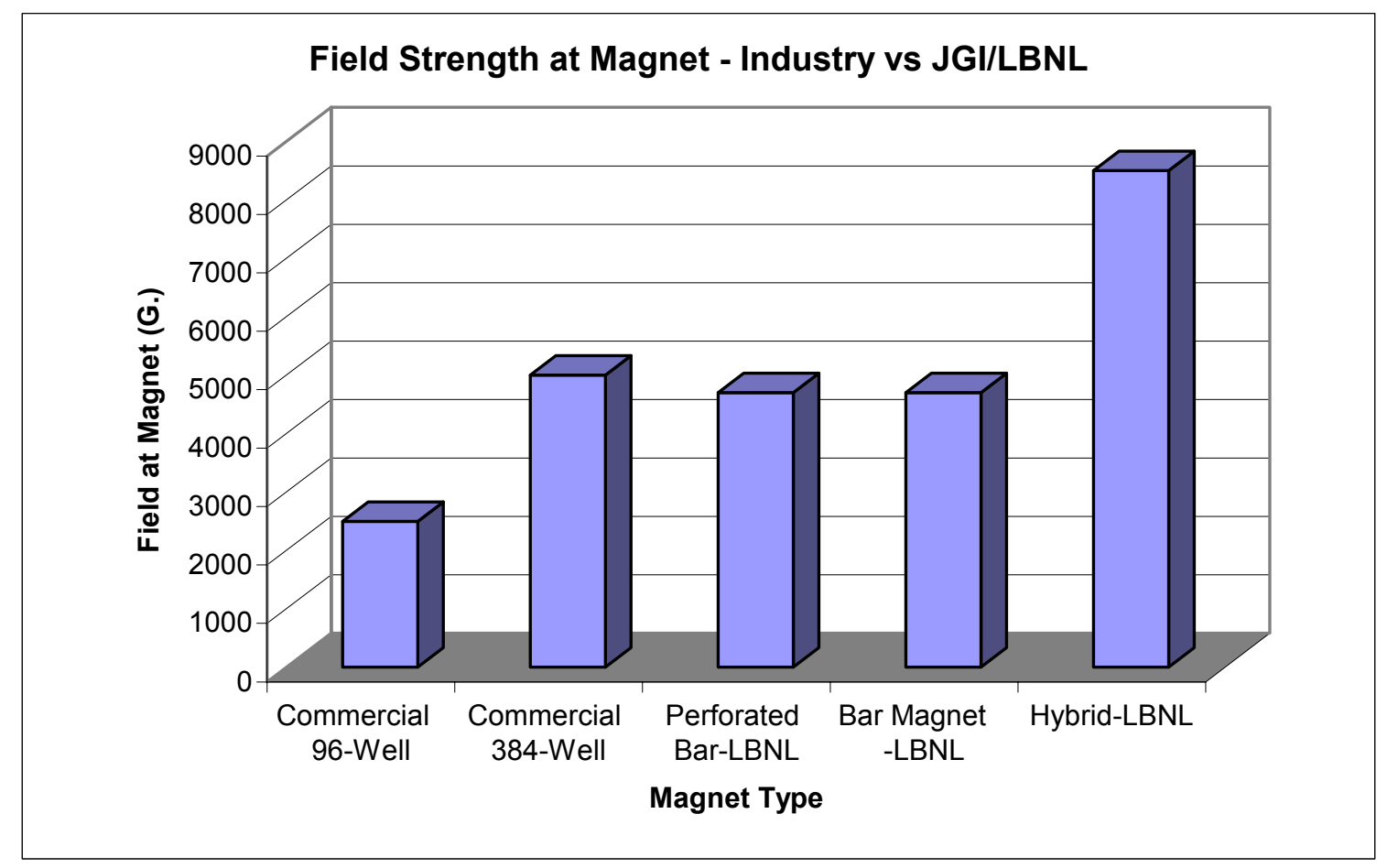

Figure 4: Field strength comparison of five magnet plates at magnet surface.

NOTE: As of Jan. 2005, the latest Hybrid magnets are exhibiting peak fields of 11000.0 Gauss. - D.H.

More importantly, as shown in figure 5, the fields at a distance of $1 \mathrm{~cm}$ above the magnet are more than $400 \%$ stronger than those of the 384-well commercial magnet and more than $1000 \%$ stronger than the fields of the 96-well commercial magnet. Thus, the range of the fields above the hybrid magnet plates is significantly greater than that of available commercial magnet plates. This aspect of the hybrid allows it to exert a much stronger force on magnetized entities that are higher above the magnetic structure, e.g., magnetized DNA or proteins that are in the upper reaches of the liquid samples in the microtiter plate wells.

The higher magnetic fields of the hybrid structures result in greater holding forces on magnetized entities that are being processed. This allows for more vigorous washing and sample volume recovery. The magnetized entities are also drawn out of solution with a higher yield and at a faster rate. Current development versions of these hybrid plates have exhibited maximum fields in excess of 9000.0 Gauss. In addition, the current design is easily modified to produce fields well above 1.0 tesla or 10000.0 gauss. 


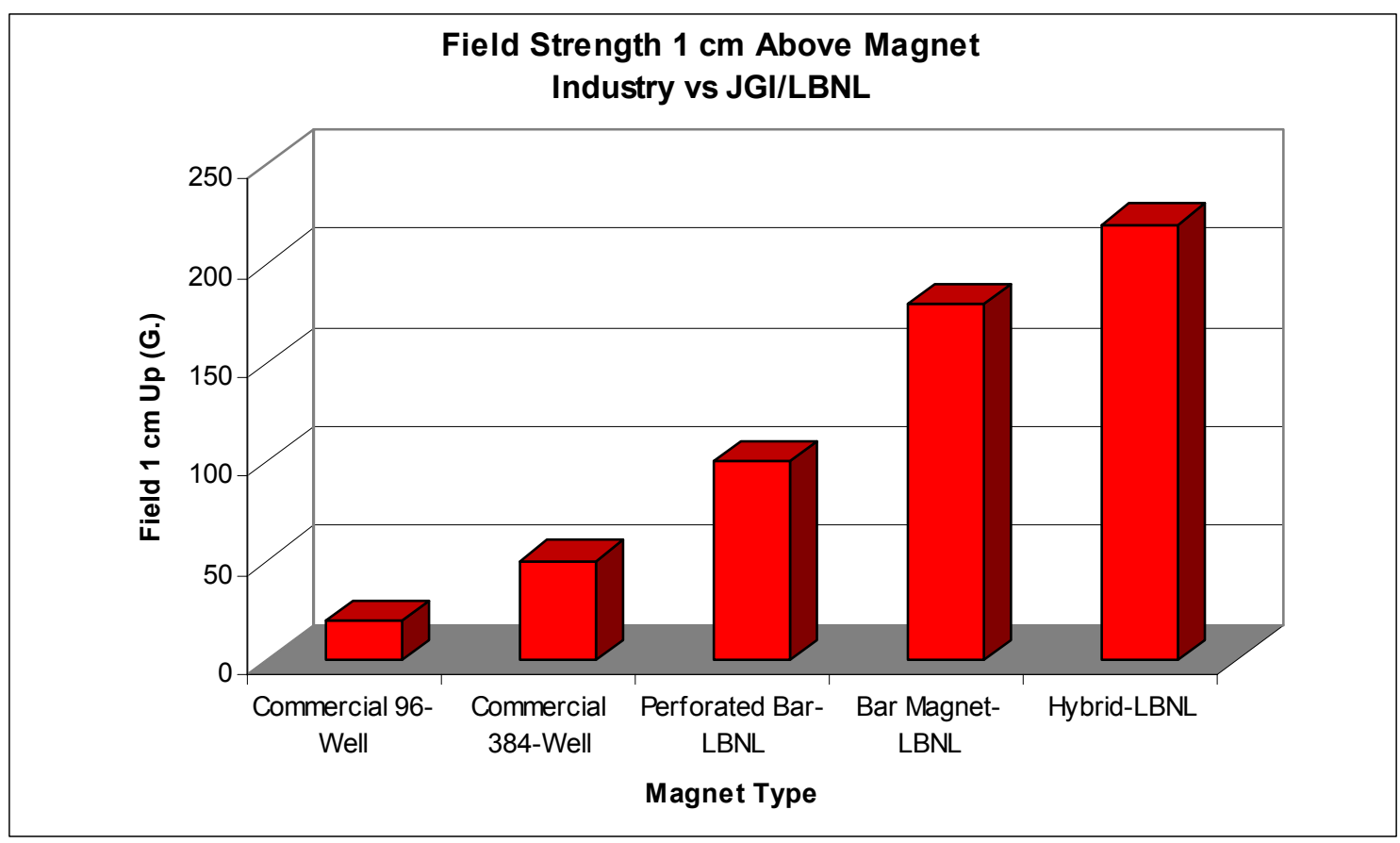

Figure 5: Field strength comparison at $1 \mathrm{~cm}$ above magnet surface.

\section{$\underline{\text { Hybrid concept }}$}

There are two common types of magnet materials which fall into the categories of permanent magnets and ferromagnetic materials.

\section{Permanent Magnets}

Permanent magnets are anisotropic or "oriented" materials which have a preferred magnetization axis. When they are magnetized they produce magnetic fields that are always "on". The distribution of these fields is dependent upon the "orientation" of the material, its geometry and other material properties. Permanent magnets are a sub-category of paramagnetic materials in that they will evidence a slight increase in magnetization when they are immersed in an external (or ambient) magnetic field. This effect is not pertinent to the applications at hand.

To date available commercial magnet plates have been "permanent magnet dominated" systems. This means that the field distributions are controlled by the geometry and orientations of the permanent magnets that are in the plates.

\section{Ferromagnetic Materials}

Ferromagnetic materials are isotropic or non-oriented. When they have not been exposed to an external magnetic field they produce no magnetic field of their own. (They will not stick to your refrigerator.) These materials include pure iron, common carbon steel alloys and more exotic materials such as vanadium permendur which is composed of iron, cobalt and vanadium. The importance of these materials is that they will tend to concentrate and redirect magnetic flux from other sources such as electromagnetic coils or permanent magnets. 


\section{Hybrid structures}

A comprehensive theory of hybrid structures was formulated by Dr. Klaus Halbach in the 1980's for accelerator applications [1]. The hybrid magnets developed at LBNL/JGI use this theory as a basis for further development. They incorporate both permanent magnets and ferromagnetic steel. The permanent magnets act as magnetic flux generators and the ferromagnetic poles act as concentrators to produce higher fields with distributions that are more easily controlled. This is called an "iron-dominated" system, i.e., the field distributions in the regions of interest are primarily controlled by the ferromagnetic pole geometry and material characteristics rather than the permanent magnets. The fields of the JGI hybrid magnets are both stronger and extend farther into the region of interest than those of currently available commercial magnet plates. They also have a more beneficial field distribution for a number of important applications.

\section{Computer modeling}

Two and three dimensional computer models were constructed to further develop and quantify the performance of these magnetic structures. The AMPERES code [3] was used to construct and solve three dimensional boundary element models (B.E.M.) that incorporated all significant geometric attributes and non-linear behavior of isotropic, ferromagnetic steel.

\section{2-D Modeling of Magnetic Structures}

The field plot shown in figure 6 is a 2-D boundary value model solved by the code PANDIRA which is a member of the POISSON SUPERFISH codes. [4] The left side of the model is a Dirichlet boundary and implies mirror image symmetry. The model shown has a geometric periodicity of $0.9 \mathrm{~cm}$. The magnetic periodicity is twice that or $1.8 \mathrm{~cm}$. Because of the left hand Dirichlet symmetry boundary, the model is a complete representation of an infinitely long structure having three full magnetic periods. The open boundaries at the right of the structure allow complete modeling of the truncation or end-effect fields.

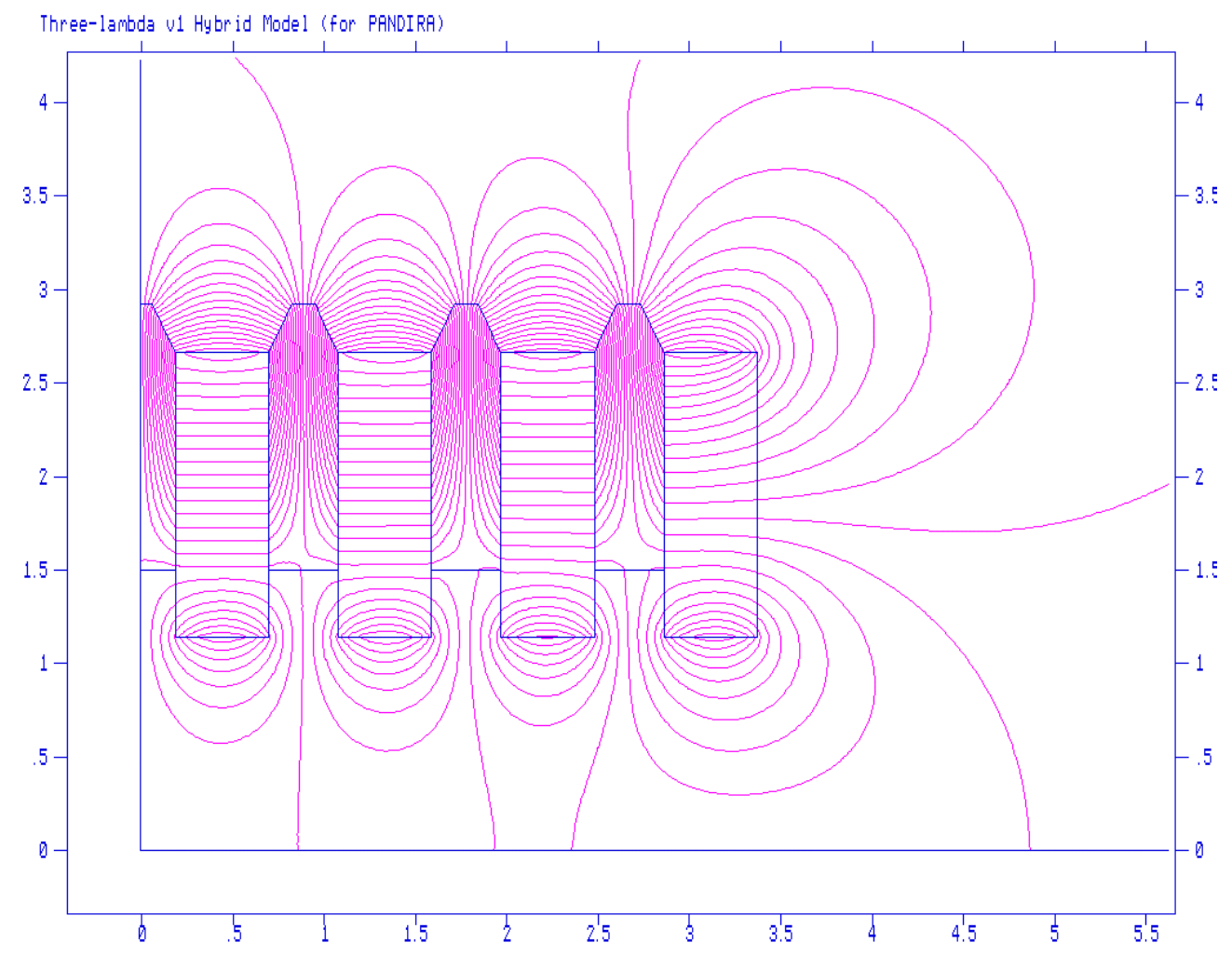

Figure 6: 2-D Pandira model with symmetry plane at left side. 
The field lines shown in figure $\mathrm{xx}$ are lines of constant vector potential A. From Maxwell, $\mathbf{B}=\mathrm{Curl} \mathbf{A}$ where $\mathbf{B}$ is the magnetic flux density and the Curl of $\mathbf{A}$ is given by:

Curl $A=\nabla \times A=\left(\frac{\partial A_{z}}{\partial y}-\frac{\partial A_{y}}{\partial z}\right) \bar{x}+\left(\frac{\partial A_{x}}{\partial z}-\frac{\partial A_{z}}{\partial x}\right)(\hat{y})+\left(\frac{\partial A_{x}}{\partial x}-\frac{\partial A_{y}}{\partial y}\right)(\hat{z})$

i.e., the cross product of the partial derivatives with respect to $\mathrm{x}, \mathrm{y}$ and $\mathrm{z}$ and the 3 -space vector quantity $\mathbf{A}$.

An implication of this relationship between the vector potential $\mathbf{A}$ and the magnetic flux density $\mathbf{B}$ is that the proximity or density of the field lines is an indication of the relative strength of the field. An increasing density of field lines can be seen moving from the bottom of the ferromagnetic poles to the trapezoidal pole tip area.

The fields in the poles range from several thousand gauss at the bottom to approximately seventeen thousand gauss in the upper corners of the trapezoidal tip. The fields in the air outside the pole tip are correspondingly high in the region of interest for magnetic separation applications. In addition, because of the geometry and polarity of the pole tip array, high field gradients are produced in the region above the pole tips.

This mechanism is central to the high performance of these magnetic structures. The force exerted on the ferrimagnetic beads attached to target molecules in a typical separation process is directly proportional to the product of the $\mathbf{B}$ field magnitude and the gradient of the $\mathbf{B}$ field. These hybrid structures produce both high fields and strong gradients.

\section{Gradient distributions}

The field gradient structure is created by the combination of linear permanent magnets and ferromagnetic steel poles. The gradient distributions of these hybrid structures can be controlled and shaped to produce both vertical and horizontal, finely-structured gradients with corresponding directional forces. This can allow, for example, magnetized entities to be strongly held at higher elevations in micro-titer plate wells for more effective separation and extraction.

\section{Grad of B:}

The gradient of the magnetic flux density $\mathbf{B}$ where $\mathbf{B}$ is a vector quantity in 3-space is given by:

$$
\operatorname{Grad} B=\nabla B=\frac{\partial B}{\partial x} \widehat{x}+\frac{\partial B}{\partial y} \widehat{y}+\frac{\partial B}{\partial z} \widehat{z}
$$

i.e., the sum of the products of the partial derivatives of $B$ with respect to $\mathrm{x}, \mathrm{y}$ and $\mathrm{z}$ and the unit vectors $\hat{x}, \hat{y}$ and $\hat{z}$.

The magnitude of the gradient of $\mathbf{B}$ is given by:

$$
|\nabla B|=\left[\left(\left(\frac{\partial B}{\partial x}\right)^{2}+\left(\frac{\partial B}{\partial y}\right)^{2}+\left(\frac{\partial B}{\partial z}\right)^{2}\right)\right]^{1 / 2}
$$


i.e., the square root of the sum of the squares of the partial derivatives of $\mathbf{B}$ with respect to $\mathrm{x}, \mathrm{y}$ and $\mathrm{z}$. The force $F_{\nabla}$ experienced by magnetized targets in the field, is proportional to the product, called the "forcedensity", of the field magnitude and the magnitude of the gradient of the field at the location of the target, i.e.,

$$
F_{\nabla} \propto|B||\nabla B|
$$

\section{Force-density distribution above pole}

The graph of figure 7 below shows the force density distribution above a single pole of a production 384well hybrid magnet structure. The pole tip is at the $y=0$ point and the graph extends to $1 \mathrm{~cm}$ above the pole tip. The average force-density along this path is $45.2 \mathrm{~T}^{\wedge} 2 / \mathrm{m}$ and the average field gradient is $92.7 \mathrm{~T} / \mathrm{m}$.

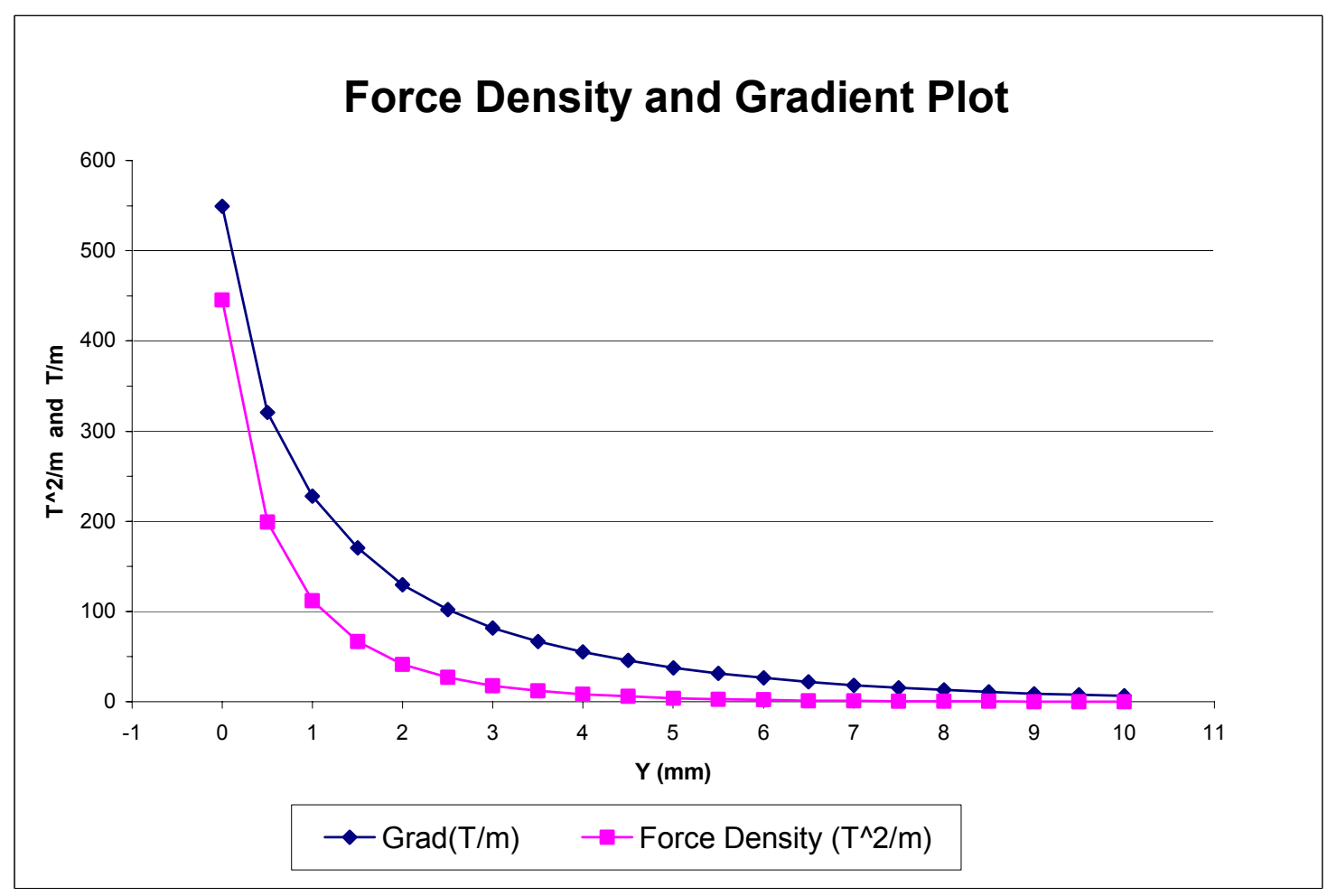

Figure 7: Force density and gradient plot from pole tip to $1 \mathrm{~cm}$ above.

\section{Variations of basic design for specialized functions}

\section{Single pole configuration}

The flux concentrating principal of the Hybrid can be used in a single-pole variant to produce a high performance non-periodic magnetic structure. Figure 8 shows a 2-D Pandira model of such a configuration. The left edge of the model is again a Dirichlet boundary and implies mirror symmetry. Hence, the magnetic structure consists of a single pole with permanent magnet material to the left and right. The device shown is scaled to produce approximately 1 tesla fields at the pole tip. The fields $2 \mathrm{~cm}$ above the pole tip are approximately 190 Gauss. 


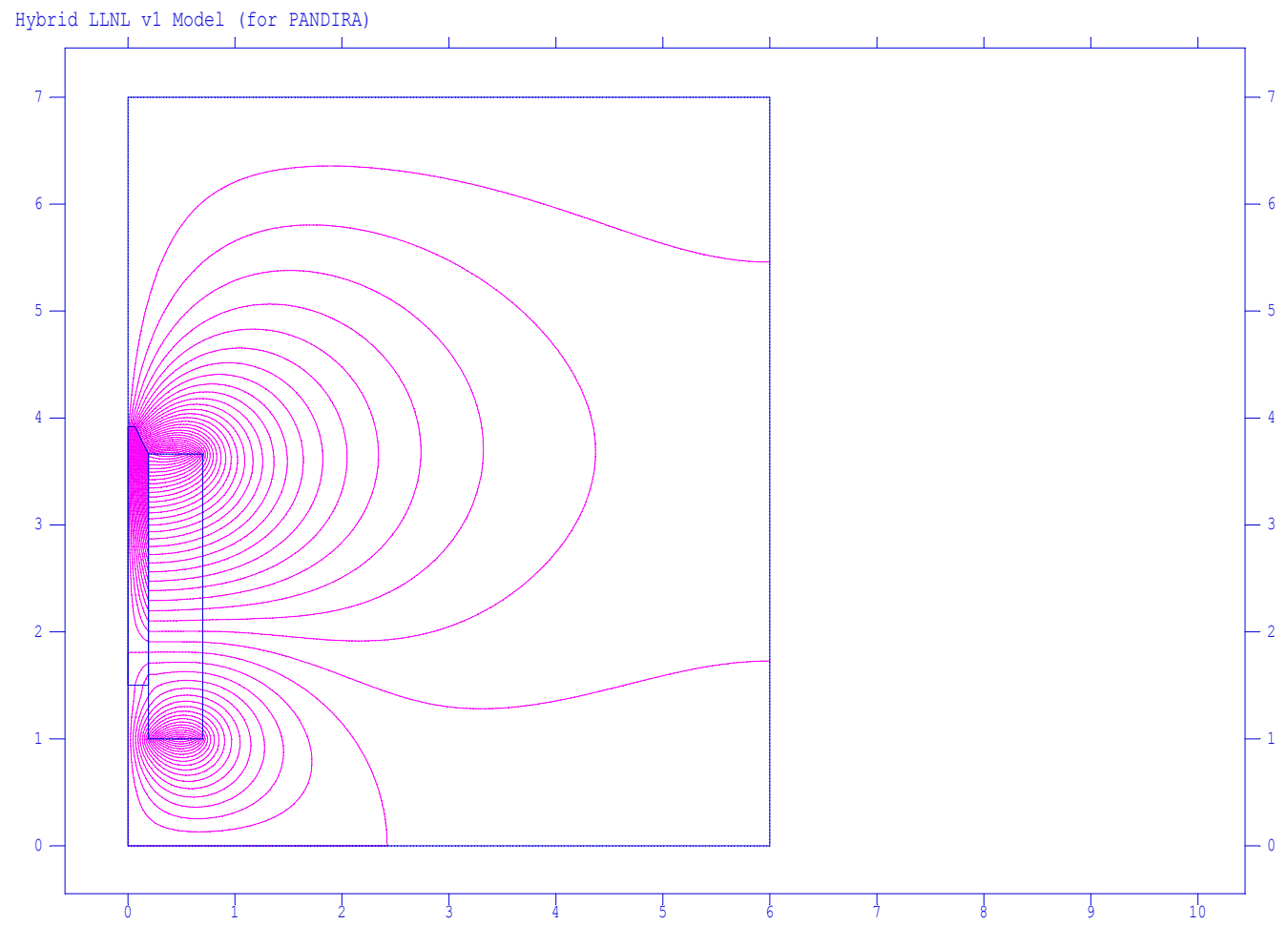

Figure 8: Single-pole variant of Hybrid structure.

\section{Radial variants}

Hybrid structures can be designed so that the poles are radially arranged to produce strong gradient distributions around cylindrical or conical vessels for target separation in either static or flow separation applications.

\section{Tee pole variants}

Poles of the hybrid structures can be easily machined to achieve complicated shapes that produce complex field distributions while maintaining high fields and strong gradients. Figure 9 below illustrates a "tee" shaped, variant cross-section of the magnet poles that produces concentrated, transverse gradient fields at elevated locations on the M-T plate wells. An array of wells is shown in relative position to the poles.

Figure 10 shows the circular cutouts in the top of the poles that conform to the well shapes of thermal cycler or "PCR" M-T plates and provide a crescent shaped, gradient force field at an arbitrary height on the well. This allows magnetized material in solution, e.g., DNA, to be held above the bottom of the wells while solutions are completely extracted by means of aspiration devices without disturbing the held, magnetized material. 


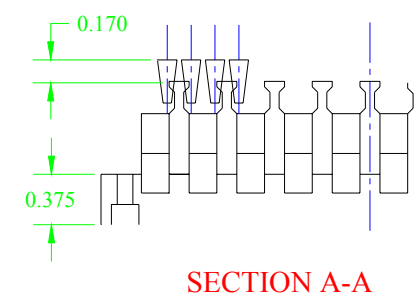

Figure 9. View showing tee shaped cross-section of poles

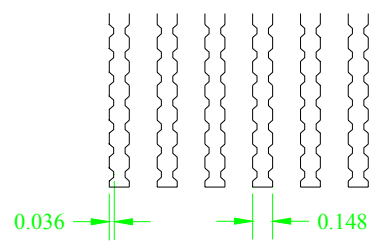

Figure 10. Top view of poles only, showing circular cut-outs for M-T plate wells

\section{$\underline{\text { Adapted to a wide range of micro-titer plates and instruments }}$}

Loading of the micro-titer plate wells is done by various instruments ranging from hand pipettors to large liquid handling robots with arrays of syringe-like devices. These magnetic structures are being adapted for use on a variety of these liquid-handling systems.

Figure 11 shows a hybrid structure assembly with lower instrument adapter plate and upper M-T plate interface. Figure 12 is an exploded view showing the interfaces more clearly. 
Lawrence Berkeley National Laboratory Report: LBNL-56017

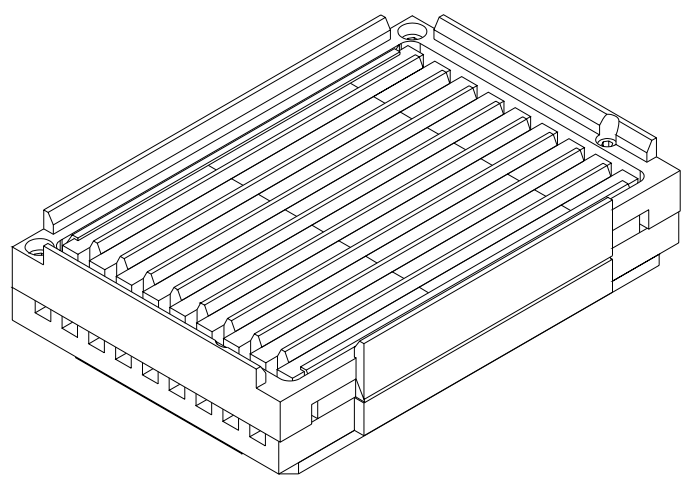

Figure 11. Hybrid magnet assembly with M-T plate interface and lower locator plate.

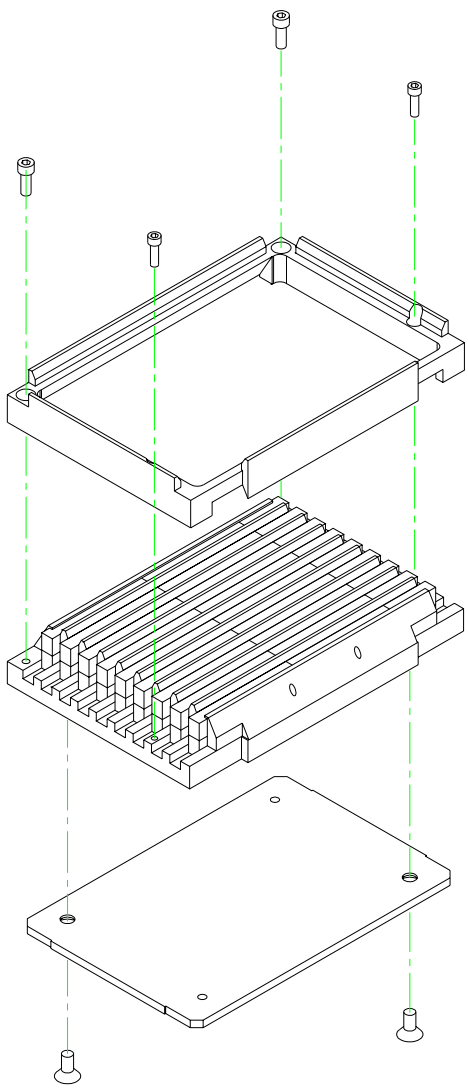

Figure 12. Exploded view of hybrid assembly with micro-titer plate interface and lower locator plate. 


\section{Conclusions}

These hybrid magnetic structures represent an enabling device that is helping to advance modern, highthroughput, production sequencing capabilities and to improve general bio-assay techniques. Their performance significantly exceeds that of currently available commercial magnet plates. In addition, the use of easy to machine, ferromagnetic poles allows for significant flexibility of design and application of these devices. Their adaptation to a range of experimental and production instruments is well under way.

\section{$\underline{\text { References }}$}

[1] K. Halbach, Insertion Device Design: 16 Lectures Presented from October 1988 to March 1989, LBL Publication V 8811-1.1-16.

[2] Christopher Elkin, Hitesh Kapur, Troy Smith, David Humphries, Martin Pollard, Nancy Hammon, Trevor Hawkins, Magnetic Bead Purification of Labeled DNA Fragments for High-Throughput Capillary Electrophoresis Sequencing, BioTechniques 32:1302, June 2002.

[3] Integrated Engineering Software, AMPERES, Three-dimensional Magnetic Field Solver , 300 Cree Crescent, Winnipeg, Manitoba, R3J 3W9, Canada, 2001.

[4] J. Billen, L. Young, POISSON SUPERFISH, LA-UR-96-1834, Univ. of California-Los Alamos National Laboratory, 2001.

[5] D. Humphries, et al, Hybrid Magnetic Structure Bonding Procedure, Engineering Note M8052, Lawrence Berkeley National Laboratory, November 2001.

\section{DISCLAIMER}

This document was prepared as an account of work sponsored by the United States Government. While this document is believed to contain correct information, neither the United States Government nor any agency thereof, nor The Regents of the University of California, nor any of their employees, makes any warranty, express or implied, or assumes any legal responsibility for the accuracy, completeness, or usefulness of any information, apparatus, product, or process disclosed, or represents that its use would not infringe privately owned rights. Reference herein to any specific commercial product, process, or service by its trade name, trademark, manufacturer, or otherwise, does not necessarily constitute or imply its endorsement, recommendation, or favoring by the United States Government or any agency thereof, or The Regents of the University of California. The views and opinions of authors expressed herein do not necessarily state or reflect those of the United States Government or any agency thereof or The Regents of the University of California. 